\title{
Salt tolerance and regulation of gas exchange and hormonal homeostasis by auxin-priming in wheat
}

\author{
Muhammad Iqbal(1) and Muhammad Ashraf(2) \\ (1)Government College University Faisalabad, Department of Botany, Faisalabad 38000, Pakistan. E-mail: iqbaluaf@yahoo.com (2)University \\ of Agriculture Faisalabad, Department of Botany, Faisalabad 38040, Pakistan. E-mail: ashrafbot@yahoo.com
}

\begin{abstract}
The objective of this work was to assess the regulatory effects of auxin-priming on gas exchange and hormonal homeostasis in spring wheat subjected to saline conditions. Seeds of MH-97 (salt-intolerant) and Inqlab-91 (salt-tolerant) cultivars were subjected to 11 priming treatments (three hormones $\mathrm{x}$ three concentrations + two controls) and evaluated under saline $\left(15 \mathrm{dS} \mathrm{m}^{-1}\right)$ and nonsaline $\left(2.84 \mathrm{dS} \mathrm{m}^{-1}\right)$ conditions. The priming treatments consisted of: $5.71,8.56$, and $11.42 \times 10^{-4} \mathrm{~mol} \mathrm{~L}^{-1}$ indoleacetic acid; $4.92,7.38$, and $9.84 \times 10^{-4} \mathrm{~mol} \mathrm{~L}^{-1}$ indolebutyric acid; 4.89, 7.34, and $9.79 \times 10^{-4} \mathrm{~mol} \mathrm{~L}^{-1}$ tryptophan; and a control with hydroprimed seeds. A negative control with nonprimed seeds was also evaluated. All priming agents diminished the effects of salinity on endogenous abscisic acid concentration in the salt-intolerant cultivar. Grain yield was positively correlated with net $\mathrm{CO}_{2}$ assimilation rate and endogenous indoleacetic acid concentration, and it was negatively correlated with abscisic acid and free polyamine concentrations. In general, the priming treatment with tryptophan at $4.89 \times 10^{-4} \mathrm{~mol} \mathrm{~L}^{-1}$ was the most effective in minimizing yield losses and reductions in net $\mathrm{CO}_{2}$ assimilation rate, under salt stress conditions. Hormonal homeostasis increases net $\mathrm{CO}_{2}$ assimilation rate and confers tolerance to salinity on spring wheat.
\end{abstract}

Index terms: Triticum aestivum, hormonal balance, plant growth regulators, salinity, seed priming, stomatal regulation.

\section{Tolerância ao sal e regulação de trocas gasosas e da homeostase hormonal pelo condicionamento osmótico com auxinas em trigo}

Resumo - O objetivo deste trabalho foi avaliar os efeitos regulatórios do condicionamento osmótico com auxinas sobre as trocas gasosas e a homeostase hormonal em trigo de primavera, submetido a condições salinas. Sementes das cultivares MH-97 (intolerante ao sal) e Inqlab-91 (tolerante ao sal) foram submetidas a 11 tratamentos de condicionamento osmótico (três hormônios x três concentrações + dois controles) e avaliadas sob condições salinas $\left(15 \mathrm{dS} \mathrm{m}^{-1}\right)$ e não salinas $\left(2,84 \mathrm{dS} \mathrm{m}^{-1}\right)$. Os tratamentos com condicionamento consistiram de: ácido indolacético a

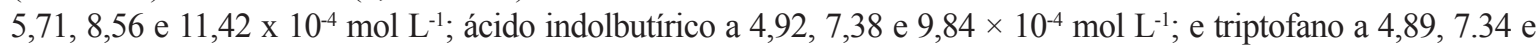
$9,79 \times 10^{-4} \mathrm{~mol} \mathrm{~L}^{-1}$; e sementes condicionadas em água (controle). Um tratamento com sementes não condicionadas também foi avaliado. Os agentes condicionadores diminuíram os efeitos de salinidade sobre a concentração de ácido abcísico endógeno, na cultivar intolerante ao sal. A produtividade de grãos correlacionou-se positivamente à taxa de assimilação líquida de $\mathrm{CO}_{2}$ e à concentração de ácido indolacético; e negativamente ao ácido abcísico e às concentrações de poliaminas livres. De forma geral, o tratamento com triptofano a $4,89 \times 10^{-4} \mathrm{~mol} \mathrm{~L}^{-1}$ foi o mais efetivo em amenizar perdas produtivas e reduções na assimilação líquida de $\mathrm{CO}_{2}$, sob condições de estresse salino. A homeostase hormonal aumentou a taxa de assimilação líquida de $\mathrm{CO}_{2}$ e conferiu tolerância à salinidade ao trigo de primavera.

Termos para indexação: Triticum aestivum, balanço hormonal, reguladores de crescimento, salinidade, condicionamento de sementes, regulação estomatal.

\section{Introduction}

Soil salinity is one of the biggest threats to global agriculture. A number of strategies have been used to overcome the salinity problem either by management or by inducing salt tolerance in plant (Flowers, 2004; Cuartero et al., 2006). Nonetheless, due to the genetic and physiological complexities of the salt tolerance trait (Flowers, 2004), the strategies to cope with salinity problem have come across a limited success. Furthermore, consumers are reluctant to accept transgenically modified food (Munns, 2005). In this scenario, seed priming has gained much popularity in inducing salt tolerance in relatively less salt-tolerant plants, since it is an easy technique, with low risk and low cost. Beneficial effects of seed priming have been

Pesq. agropec. bras., Brasília, v.48, n.9, p.1210-1219, set. 2013

DOI: 10.1590/S0100-204X2013000900004 
reported for many crops; however, its mechanisms, particularly at later growth stages of plants, remain largely unknown.

Plant hormones affect a wide spectrum of processes in plants under both normal and stressful environments. Exogenous application of plant growth regulators (PGR) have been shown to alleviate the drastic effects of abiotic stresses on germination and plant growth (Finch-Savage et al., 2004; Javid et al., 2011). Different stresses alter the endogenous levels of hormones, and thus affect plant growth. Therefore, plant response to salinity is not exclusively a function of the osmotic shock or ionic toxicity, but also appears to be a consequence of the effect of salt on hormonal balance.

In contrast to abscisic acid, auxins are mainly synthesized in the shoots. Although indoleacetic acid (IAA) biosynthesis follows several pathways, its most common precursor is tryptophan (TRP). Seed priming with auxins has been shown to alleviate the inhibitory effects of salt stress on germination and growth in wheat (Iqbal \& Ashraf, 2007). Relatively very little, and often confusing information are available concerning the involvement of auxins in stomatal regulation (Pospíšilová, 2003). For instance, Assmann \& Armstrong (1999) found a differential effect of low and high IAA concentration on stomatal conductance $\left(\mathrm{g}_{\mathrm{s}}\right)$, whereas, in Pisum sativum and Phaseolus vulgaris, Eamus (1986) found $\mathrm{CO}_{2}$ concentration-dependent effect of IAA on $\mathrm{g}_{\mathrm{s}}$. Auxin is known to antagonize abscisic acid (ABA) induced stomatal closure in Arabidopsis (Tanaka et al., 2006). Ethylene has been shown to mediate the auxin-induced stomatal opening in some other plants (Merritt et al., 2001; Iqbal et al., 2011). Therefore, changes in IAA concentrations might regulate stomatal opening and closing in a concentration-dependent manner.

In comparison with the information on endogenous hormonal contents in plant tissues, information still lack on the auxin-priming-induced changes in endogenous hormonal concentrations in plants under salt stress. Therefore, it is important to study the effects of natural auxins and their precursors (TRP) as priming agents on the endogenous content of auxins. Moreover, ABA and polyamines could provide valuable information on their mechanism of action on plant growth.

The objective of this work was to assess the regulatory effects of auxin-priming on gas exchange and hormonal homeostasis in spring wheat subjected to saline conditions.

\section{Materials and Methods}

Field experiments were conducted during the winter 2002 and 2003, in the Botanic Garden, University of Agriculture, Faisalabad, where the average photosynthetically active radiation (PAR) of the entire growth period was $1,098 \mu \mathrm{mol} \mathrm{m}^{-2} \mathrm{~s}^{-1}$, and the maximum and minimum relative humidity was 79 and $32 \%$, respectively. The average maximum and minimum temperatures during the growth period were $28 \pm 4^{\circ} \mathrm{C}$ and $12 \pm 3^{\circ} \mathrm{C}$, respectively.

Seeds (17 g) of two spring wheat cultivars, MH-97 (salt intolerant) and Inqlab-91 (salt tolerant) were subjected to 11 priming treatments (three hormones $\mathrm{x}$ three concentrations +2 controls) and evaluated in saline $\left(150 \mathrm{mmol} \mathrm{L}^{-1} \mathrm{NaCl}\right)$ and nonsaline conditions. Seeds were primed separately, imbibed for 12 hours in solutions of: 5.71, 8.56, and $11.42 \times 10^{-4} \mathrm{~mol} \mathrm{~L}^{-1} \mathrm{IAA} ; 4.92,7.38$, and $9.84 \times 10^{-4} \mathrm{~mol} \mathrm{~L}^{-1} \mathrm{IBA}$; and 4.89, 7.34, and $9.79 \times 10^{-4} \mathrm{~mol} \mathrm{~L}^{-1} \mathrm{TRP}$, as well as in distilled water (control). A negative control without any kind of priming was also evaluated. After pre-soaking, seeds were surface-dried with filter paper and then air-dried for 12 hours at room temperature. Seeds were sown in seedbeds (732x137x46 cm of length, width and depth, respectively) lined with polythene sheets and filled with thoroughly mixed sandy loam soil (saturation percentage $=25.5 ; \mathrm{EC}_{\mathrm{e}}=2.84 \mathrm{dS} \mathrm{m}{ }^{-1} ; \mathrm{pH}=7.56$ ). It was used a randomized block design, arranged in split-split plots (hormones in the main plots, saline conditions in the subplots, and cultivars in the sub-subplots), with four replicates. After three weeks, the primed (air-dried for 12 hours) and nonprimed seeds of both cultivars, were sown in the soil having moisture contents $13 \%$ (Iqbal \& Ashraf, 2007), keeping row-to-row spacing about $15 \mathrm{~cm}$. Plants were irrigated carefully with tap water $\left(\mathrm{EC}_{\mathrm{w}}=0.65\right.$ $\mathrm{dS} \mathrm{m}^{-1}$ ), so as to minimize dilution in salinity at the irrigation points. Electrical conductivity of the soil was checked periodically and maintained whenever required.

At boot stage, 12 plants from each treatment (three plants per replicate) were uprooted, washed with distilled water, and analyzed for shoot fresh biomass. At maturity, four plants per replicate were used to record plant height, number of fertile tillers per plant, and components of grain yield.

Measurements of net $\mathrm{CO}_{2}$ assimilation rate (A), transpiration rate $(\mathrm{E})$, and stomatal conductance $\left(\mathrm{g}_{\mathrm{s}}\right)$ were made from 10:00 to 15:00 $\mathrm{h}$ at the boot stage (four plants per replicate) on a recently matured top leaf, using an open system LCA-4 ADC portable infrared gas 
analyzer (Analytical Development Company, Hoddesdon, England). Measurements were made with the following specifications/adjustments: leaf chamber area, $11.35 \mathrm{~cm}^{2}$;

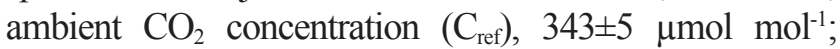
substomatal $\mathrm{CO}_{2}$ concentration $\left(\mathrm{C}_{\mathrm{i}}\right), 235 \pm 34 \mu \mathrm{mol} \mathrm{mol}{ }^{-1}$; leaf chamber temperature $\left(\mathrm{T}_{\mathrm{ch}}\right), 39 \pm 4^{\circ} \mathrm{C}$; leaf chamber gas flow rate $(\mathrm{V}), 389 \pm 3 \mathrm{~mL} \mathrm{~min}^{-1}$; molar flow of air per unit leaf area $\left(\mathrm{U}_{\mathrm{s}}\right), 223 \pm 1.6 \mathrm{~mol} \mathrm{~m}^{-2} \mathrm{~s}^{-1}$; ambient pressure (P), 99.8 $\pm 0.7 \mathrm{kPa}$; and light intensity at leaf surface $\left(\mathrm{Q}_{\text {leaf }}\right)$, which was up to $1,025 \pm 72 \mu \mathrm{mol} \mathrm{m}^{-2} \mathrm{~s}^{-1}$.

Recently matured top leaves were used for the determination of different phytohormones at boot stage. Auxins (IAA, IBA) and ABA were extracted and purified as described by Kusaba et al. (1998), with some modifications (Iqbal et al., 2006). The quantification of plant hormones was done by high performance liquid chromatograph (HPLC) using authentic standards run through for the whole procedure, as previously described (Iqbal et al., 2006).

Fresh, recently matured top leaves $(2 \mathrm{~g})$ were sampled at boot stage, and ground in $20 \mathrm{~mL}$ of cold $5 \%(\mathrm{v} / \mathrm{v})$ aqueous perchloric acid. For polyamines (putrescine, PUT; spermidine, SPD and spermine, SPM) extraction and quantification, benzoylation method was performed as previously described (Flores \& Galston, 1982), with minor modifications.

Collected data were analysed using the GLM module of CoStat version 6.2 (CoHort Software, Monterey, CA, USA). Analysis of variance was done using a randomized complete block design with split-split plot arrangement (d.f. = 120). Correlations among grain yield, gas exchange characteristics, and hormonal concentrations were determined using XLSTAT version 7.5.2 (Addinsoft SARL, Paris, FR). Means were compared using LSD values calculated according to Duncan's multiple range test, at $5 \%$ probability.

\section{Results and Discussion}

Higher concentrations of the priming agents (IAA, IBA and TRP) increased plant height in the salt-tolerant cultivar (Table 1). However, the lowest concentration $\left(4.89 \times 10^{-4} \mathrm{~mol} \mathrm{~L}^{-1}\right)$ of TRP was much effective in increasing plant height in the salt-intolerant cultivar. Priming agents had nonsignificant effects on shoot fresh biomass, compared to the control. Plants raised from seeds primed at low concentration of TRP, in the salt-tolerant cultivar, and in high concentration of IBA in the salt-intolerant cultivar, had more fertile tillers than the control. IAA is present in plants mostly in the form of IAA conjugates (Ludwig-Müller, 2011). Thus, the better growth induced by TRP could be attributed to TRP-dependent synthesis of free IAA, particularly at early seedling stage. Exogenous application of auxin has already been shown to increase growth in wheat under salt stress (Iqbal \& Ashraf, 2007), and in barley under water stress (Ashraf et al., 2006).

Low concentration $\left(4.89 \times 10^{-4} \mathrm{~mol} \mathrm{~L}^{-1}\right)$ of TRP was much effective in increasing $\mathrm{A}$ (net $\mathrm{CO}_{2}$ assimilation rate) in the two cultivars, under both saline and nonsaline conditions (Figure 1). Although the priming agents did not affect $g_{s}$ (stomatal conductance) in the salt-tolerant cultivar, they significantly increased $g_{s}$ in the salt-intolerant cultivar. The results suggested that TRP-dependent IAA synthesis might be involved in enhancing A through stomatal opening, under both control and salt stress. The auxin-mediated enhancement in $\mathrm{g}_{\mathrm{s}}$ and A was already reported for Vicia faba (Merritt et al., 2001) and Hordeum vulgare (Ashraf et al., 2006). Exogenous application of TRP increased photosynthetic pigments (chlorophyll a and b) in wheat under salt stress (El-Bassiouny, 2005).

Seed priming in TRP $\left(4.89 \times 10^{-4} \mathrm{~mol} \mathrm{~L}^{-1}\right)$ increased $\mathrm{E}$ (transpiration rate) in the salt-intolerant cultivar, while all priming agents increased $\mathrm{E}$ in the salt-stressed plants of the salt-tolerant cultivar, when compared with the control. Interestingly, soil salinity reduced much more $\mathrm{E}$ in the salt-tolerant than in the salt-intolerant wheat cultivar. The reduction in $\mathrm{E}$ is considered an important adaptive mechanism of salinity-tolerance in wheat (Robinson, 1988). This mechanism causes reduction in salt uptake, which usually occurs passively in wheat through the transpiration stream (Yeo \& Flowers, 1986). Although the salt-tolerant cultivar had more $g_{s}$ than the salt-intolerant one, it exhibited lower E under salt stress. These results suggest that salt-tolerant cultivar has a better control over the stomata, under salt stress.

James et al. (2002) observed reduction in $g_{s}$ before any apparent decline in leaf water potential in the salt-stressed wheat plants. Similarly, Sadeghi \& Nazemosadat (2011) observed substantial decrease in $g_{s}$ before any noticeable change in leaf water potential in tolerant wheat lines. In this situation, enhanced transport of photoassimilates towards developing seeds and reduced utilization of photoassimilates in the vegetative organs could have compensated the reduction of yield in the salt-tolerant wheat cultivar (Grieve et al., 1992; Iqbal \& Ashraf, 2007). In cotton, foliar spray with IAA partially counteracted the effect of water deficit on $\mathrm{g}_{\mathrm{s}}$, A and $\mathrm{E}$ (Kumar et al., 2001). In contrast, IBA did not affect $\mathrm{E}$ and $\mathrm{A}$ in either wild type 
or ABA-deficient mutants of tomato (Herde et al., 1997). Taken together, the results suggest that TRP-priming removed both stomatal and nonstomatal limitations, and thus increased A in genetically diverse wheat cultivars.
Priming agents did not increase free IAA concentrations in the leaves, in the salt-intolerant cultivar, in comparison with the control (Table 2). Generally, auxin biosynthesis (Normanly, 2010), auxin influx and efflux carriers (Krupinski

Table 1. Growth parameters of wheat plants (mean $\pm \mathrm{SE}, \mathrm{n}=4)$ at $2.8 \mathrm{dS} \mathrm{m}^{-1}$ (control) and $15.0 \mathrm{dS} \mathrm{m} \mathrm{m}^{-1}(\mathrm{salt})$, raised from primed seeds with indoleacetic acid, indolebutyric acid, and tryptophan solutions ${ }^{(1)}$.

\begin{tabular}{|c|c|c|c|c|}
\hline \multirow[t]{2}{*}{ Pre-sowing seed treatments } & \multicolumn{2}{|c|}{ Salt-intolerant (MH-97) } & \multicolumn{2}{|c|}{ Salt-tolerant (Inqlab-91) } \\
\hline & Control & Salt & Control & Salt \\
\hline & \multicolumn{4}{|c|}{ Plant height $(\mathrm{cm})$} \\
\hline \multicolumn{5}{|l|}{ Indoleacetic acid } \\
\hline $5.71 \times 10^{-4} \mathrm{~mol} \mathrm{~L}^{-1}$ & $82.3 \pm 1.42$ & $61.0 \pm 1.27$ & $75.2 \pm 1.39$ & $62.8 \pm 1.20$ \\
\hline $8.56 \times 10^{-4} \mathrm{~mol} \mathrm{~L}^{-1}$ & $82.9 \pm 1.46$ & $67.8 \pm 1.81$ & $74.4 \pm 1.41$ & $65.2 \pm 1.34$ \\
\hline $11.4 \times 10^{-4} \mathrm{~mol} \mathrm{~L}^{-1}$ & $80.3 \pm 1.52$ & $63.9 \pm 1.32$ & $76.8 \pm 1.38$ & $66.3 \pm 1.29$ \\
\hline \multicolumn{5}{|l|}{ Indolebutyric acid } \\
\hline $4.92 \times 10^{-4} \mathrm{~mol} \mathrm{~L}^{-1}$ & $79.8 \pm 1.53$ & $59.0 \pm 1.23$ & $80.5 \pm 1.41$ & $60.2 \pm 1.31$ \\
\hline $7.38 \times 10^{-4} \mathrm{~mol} \mathrm{~L}^{-1}$ & $82.0 \pm 1.45$ & $67.8 \pm 1.39$ & $77.6 \pm 1.42$ & $64.5 \pm 1.37$ \\
\hline $9.84 \times 10^{-4} \mathrm{~mol} \mathrm{~L}^{-1}$ & $84.1 \pm 1.46$ & $69.7 \pm 1.43$ & $78.5 \pm 1.38$ & $65.3 \pm 1.52$ \\
\hline \multicolumn{5}{|l|}{ Tryptophan } \\
\hline $4.89 \times 10^{-4} \mathrm{~mol} \mathrm{~L}^{-1}$ & $77.8 \pm 1.50$ & $74.1 \pm 1.58$ & $80.0 \pm 1.46$ & $61.1 \pm 1.23$ \\
\hline $7.34 \times 10^{-4} \mathrm{~mol} \mathrm{~L}^{-1}$ & $81.2 \pm 1.55$ & $72.1 \pm 1.43$ & $82.7 \pm 1.63$ & $59.4 \pm 1.17$ \\
\hline $9.79 \times 10^{-4} \mathrm{~mol} \mathrm{~L}^{-1}$ & $82.5 \pm 1.41$ & $67.3 \pm 1.46$ & $83.9 \pm 1.52$ & $66.5 \pm 1.40$ \\
\hline Distilled water & $83.4 \pm 1.43$ & $71.6 \pm 1.47$ & $80.5 \pm 1.38$ & $58.5 \pm 1.32$ \\
\hline Untreated & $75.4 \pm 1.38$ & $57.1 \pm 1.20$ & $77.6 \pm 1.42$ & $49.9 \pm 1.13$ \\
\hline \multicolumn{5}{|l|}{ LSD 5\% $=0.704$} \\
\hline \multicolumn{5}{|c|}{ Shoot fresh weight (g) } \\
\hline \multicolumn{5}{|l|}{ Indoleacetic acid } \\
\hline $5.71 \times 10^{-4} \mathrm{~mol} \mathrm{~L}^{-1}$ & $24.1 \pm 1.68$ & $14.8 \pm 0.78$ & $26.8 \pm 2.05$ & $14.9 \pm 0.76$ \\
\hline $8.56 \times 10^{-4} \mathrm{~mol} \mathrm{~L}^{-1}$ & $24.2 \pm 1.52$ & $15.8 \pm 0.83$ & $30.6 \pm 3.08$ & $11.6 \pm 0.59$ \\
\hline $11.4 \times 10^{-4} \mathrm{~mol} \mathrm{~L}^{-1}$ & $24.3 \pm 2.03$ & $17.7 \pm 0.93$ & $32.1 \pm 3.36$ & $13.8 \pm 0.70$ \\
\hline \multicolumn{5}{|l|}{ Indolebutyric acid } \\
\hline $4.92 \times 10^{-4} \mathrm{~mol} \mathrm{~L}^{-1}$ & $26.0 \pm 1.94$ & $13.5 \pm 0.71$ & $25.3 \pm 1.76$ & $15.7 \pm 0.79$ \\
\hline $7.38 \times 10^{-4} \mathrm{~mol} \mathrm{~L}^{-1}$ & $28.6 \pm 2.79$ & $14.4 \pm 0.76$ & $26.5 \pm 1.45$ & $16.8 \pm 0.85$ \\
\hline $9.84 \times 10^{-4} \mathrm{~mol} \mathrm{~L}^{-1}$ & $25.5 \pm 2.24$ & $14.8 \pm 0.78$ & $27.2 \pm 2.22$ & $14.5 \pm 0.74$ \\
\hline \multicolumn{5}{|l|}{ Tryptophan } \\
\hline $4.89 \times 10^{-4} \mathrm{~mol} \mathrm{~L}^{-1}$ & $28.7 \pm 2.77$ & $15.5 \pm 0.79$ & $31.7 \pm 1.88$ & $13.8 \pm 0.69$ \\
\hline $7.34 \times 10^{-4} \mathrm{~mol} \mathrm{~L}^{-1}$ & $29.6 \pm 3.05$ & $17.0 \pm 0.87$ & $28.4 \pm 1.76$ & $14.1 \pm 0.72$ \\
\hline $9.79 \times 10^{-4} \mathrm{~mol} \mathrm{~L}^{-1}$ & $27.9 \pm 2.71$ & $16.5 \pm 0.89$ & $29.7 \pm 1.38$ & $14.0 \pm 0.71$ \\
\hline Distilled water & $29.8 \pm 2.09$ & $15.9 \pm 0.84$ & $24.6 \pm 2.61$ & $16.4 \pm 0.83$ \\
\hline Untreated & $27.1 \pm 1.87$ & $15.2 \pm 0.80$ & $28.9 \pm 1.71$ & $14.6 \pm 0.74$ \\
\hline \multicolumn{5}{|l|}{ LSD 5\% $=1.62$} \\
\hline \multicolumn{5}{|c|}{ Fertile tillers per plant } \\
\hline \multicolumn{5}{|l|}{ Indoleacetic acid } \\
\hline $5.71 \times 10^{-4} \mathrm{~mol} \mathrm{~L}^{-1}$ & $2.02 \pm 0.16$ & $1.67 \pm 0.26$ & $2.00 \pm 0.14$ & $1.37 \pm 0.04$ \\
\hline $8.56 \times 10^{-4} \mathrm{~mol} \mathrm{~L}^{-1}$ & $1.79 \pm 0.09$ & $1.83 \pm 0.07$ & $1.92 \pm 0.13$ & $1.20 \pm 0.08$ \\
\hline $11.4 \times 10^{-4} \mathrm{~mol} \mathrm{~L}^{-1}$ & $1.70 \pm 0.15$ & $1.58 \pm 0.16$ & $2.05 \pm 0.18$ & $1.20 \pm 0.08$ \\
\hline \multicolumn{5}{|l|}{ Indolebutyric acid } \\
\hline $4.92 \times 10^{-4} \mathrm{~mol} \mathrm{~L}^{-1}$ & $1.79 \pm 0.09$ & $1.33 \pm 0.00$ & $2.43 \pm 0.20$ & $1.20 \pm 0.08$ \\
\hline $7.38 \times 10^{-4} \mathrm{~mol} \mathrm{~L}^{-1}$ & $1.65 \pm 0.16$ & $1.54 \pm 0.08$ & $1.99 \pm 0.11$ & $1.37 \pm 0.04$ \\
\hline $9.84 \times 10^{-4} \mathrm{~mol} \mathrm{~L}^{-1}$ & $1.79 \pm 0.09$ & $2.17 \pm 0.07$ & $2.00 \pm 0.10$ & $1.37 \pm 0.04$ \\
\hline \multicolumn{5}{|l|}{ Tryptophan } \\
\hline $4.89 \times 10^{-4} \mathrm{~mol} \mathrm{~L}^{-1}$ & $2.09 \pm 0.16$ & $1.58 \pm 0.28$ & $2.05 \pm 0.11$ & $1.71 \pm 0.14$ \\
\hline $7.34 \times 10^{-4} \mathrm{~mol} \mathrm{~L}^{-1}$ & $1.65 \pm 0.16$ & $1.50 \pm 0.22$ & $2.32 \pm 0.13$ & $1.71 \pm 0.14$ \\
\hline $9.79 \times 10^{-4} \mathrm{~mol} \mathrm{~L}^{-1}$ & $2.02 \pm 0.16$ & $1.67 \pm 0.26$ & $1.98 \pm 0.15$ & $1.20 \pm 0.08$ \\
\hline Distilled water & $1.79 \pm 0.09$ & $1.21 \pm 0.08$ & $2.13 \pm 0.09$ & $1.37 \pm 0.14$ \\
\hline Untreated & $2.09 \pm 0.16$ & $1.00 \pm 0.00$ & $2.04 \pm 0.09$ & $1.08 \pm 0.05$ \\
\hline
\end{tabular}

${ }^{(1)} \mathrm{LSD}$, least significant difference; distilled water, hydropriming; untreated, nonprimed seeds. 

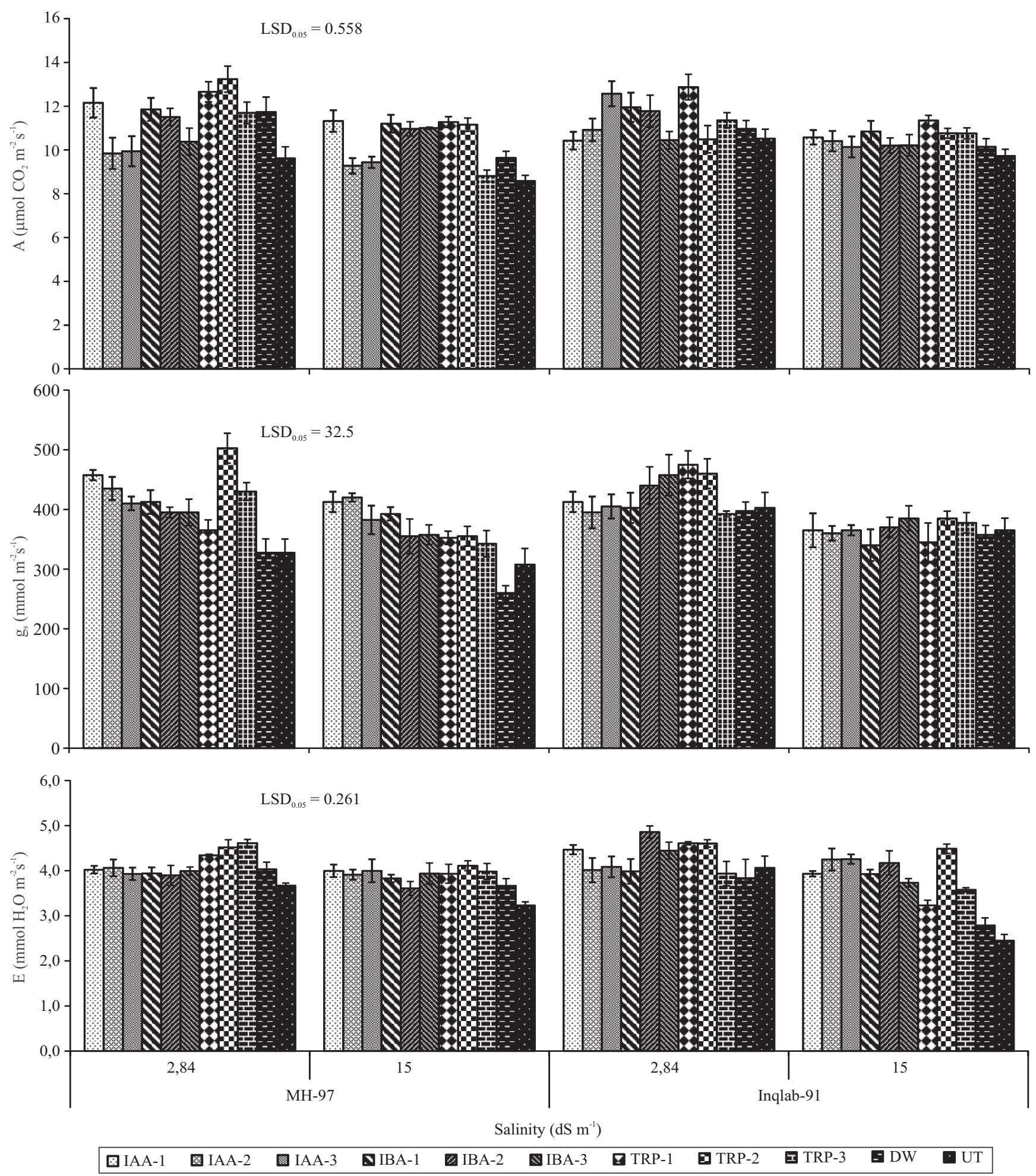

Figure 1. Gaseous exchange characteristics of wheat plants raised from primed seeds with auxins, or tryptophan, and sown under both control $\left(2.82 \mathrm{dS} \mathrm{m}^{-1}\right)$ and saline $\left(15.0 \mathrm{dS} \mathrm{m}^{-1}\right)$ conditions. IAA-1-3, indoleacetic acid (5.71, 8.56, and $11.42 \times$ $10^{-4} \mathrm{~mol} \mathrm{~L}^{-1}$, respectively); IBA-1-3, indolebutyric acid (4.92, 7.38 and $9.84 \times 10^{-4} \mathrm{~mol} \mathrm{~L}^{-1}$, respectively); TRP-1-3, tryptophan (4.89, 7.34 and $9.79 \times 10^{-4} \mathrm{~mol} \mathrm{~L}^{-1}$, respectively); DW, soaking in distilled water (hydropriming); UT, untreated (nonprimed) seeds; $\mathrm{A}$, net $\mathrm{CO}_{2}$ assimilation rate; $\mathrm{g}_{\mathrm{s}}$, stomatal conductance; $\mathrm{E}$, transpiration rate. 
\& Jonsson, 2010), as well as diverse interacting hormones affect free auxin gradients in space and time (Liu et al., 2013). In addition, the potential of plants to conjugate IAA and their capacity to hydrolyse them varies at different developmental stages, as well as under different stresses (Ludwig-Müller et al., 1996). In this context, our results suggested that auxin-priming either altered IAA biosynthesis or accelerated the IAA metabolism for conjugate formations,

Table 2. Free hormonal concentrations ( $\mathrm{ng} \mathrm{g}^{-1}$ fresh weight) in leaves of wheat plants (mean $\pm \mathrm{SE}, \mathrm{n}=4$ ), at $2.8 \mathrm{dS} \mathrm{m}^{-1}$ (control) and $15.0 \mathrm{dS} \mathrm{m}^{-1}$ (salt), raised from primed seeds with indoleacetic acid, indolebutyric acid, and tryptophan solutions ${ }^{(1)}$.

\begin{tabular}{|c|c|c|c|c|}
\hline \multirow{2}{*}{ Pre-sowing seed treatments } & \multicolumn{2}{|c|}{ Salt-intolerant (MH 97) } & \multicolumn{2}{|c|}{ Salt-tolerant (Inqlab 91) } \\
\hline & Control & Salt & Control & Salt \\
\hline & \multicolumn{4}{|c|}{ Indoleacetic acid } \\
\hline \multicolumn{5}{|l|}{ Indoleacetic acid } \\
\hline $5.71 \times 10^{-4} \mathrm{~mol} \mathrm{~L}^{-1}$ & $419.6 \pm 32.1$ & $132.2 \pm 18.6$ & $303.8 \pm 16.0$ & $145.1 \pm 08.4$ \\
\hline $8.56 \times 10^{-4} \mathrm{~mol} \mathrm{~L}^{-1}$ & $483.5 \pm 07.0$ & $114.6 \pm 11.1$ & $149.5 \pm 20.1$ & $68.9 \pm 14.7$ \\
\hline $11.4 \times 10^{-4} \mathrm{~mol} \mathrm{~L}^{-1}$ & $289.0 \pm 30.6$ & $126.2 \pm 04.0$ & $282.6 \pm 04.2$ & $150.8 \pm 24.8$ \\
\hline \multicolumn{5}{|l|}{ Indolebutyric acid } \\
\hline $4.92 \times 10^{-4} \mathrm{~mol} \mathrm{~L}^{-1}$ & $294.1 \pm 25.8$ & $159.9 \pm 32.3$ & $195.0 \pm 27.3$ & $198.8 \pm 16.2$ \\
\hline $7.38 \times 10^{-4} \mathrm{~mol} \mathrm{~L}^{-1}$ & $153.6 \pm 06.1$ & $92.7 \pm 04.9$ & $138.6 \pm 01.3$ & $116.3 \pm 30.0$ \\
\hline $9.84 \times 10^{-4} \mathrm{~mol} \mathrm{~L}^{-1}$ & $358.2 \pm 40.9$ & $154.5 \pm 11.7$ & $111.2 \pm 23.9$ & $145.6 \pm 16.6$ \\
\hline \multicolumn{5}{|l|}{ Tryptophan } \\
\hline $4.89 \times 10^{-4} \mathrm{~mol} \mathrm{~L}^{-1}$ & $251.2 \pm 03.6$ & $120.1 \pm 16.6$ & $221.3 \pm 14.2$ & $96.5 \pm 16.8$ \\
\hline $7.34 \times 10^{-4} \mathrm{~mol} \mathrm{~L}^{-1}$ & $318.4 \pm 11.9$ & $190.7 \pm 11.1$ & $266.5 \pm 01.3$ & $165.6 \pm 08.1$ \\
\hline $9.79 \times 10^{-4} \mathrm{~mol} \mathrm{~L}^{-1}$ & $216.4 \pm 30.3$ & $125.8 \pm 06.4$ & $134.9 \pm 23.6$ & $96.9 \pm 38.0$ \\
\hline Distilled water & $421.7 \pm 18.7$ & $180.9 \pm 16.6$ & $561.4 \pm 49.1$ & $88.2 \pm 01.8$ \\
\hline Untreated & $472.5 \pm 19.5$ & $138.1 \pm 35.4$ & $288.9 \pm 21.3$ & $101.5 \pm 01.6$ \\
\hline \multicolumn{5}{|l|}{ LSD 5\% $=25.6$} \\
\hline \multicolumn{5}{|c|}{ Indolebutyric acid } \\
\hline \multicolumn{5}{|l|}{ Indoleacetic acid } \\
\hline $5.71 \times 10^{-4} \mathrm{~mol} \mathrm{~L}^{-1}$ & $214.5 \pm 40.6$ & $265.2 \pm 32.5$ & $134.3 \pm 15.7$ & $94.1 \pm 27.5$ \\
\hline $8.56 \times 10^{-4} \mathrm{~mol} \mathrm{~L}^{-1}$ & $71.0 \pm 20.2$ & $252.6 \pm 39.4$ & $267.7 \pm 42.8$ & $88.9 \pm 15.4$ \\
\hline $11.4 \times 10^{-4} \mathrm{~mol} \mathrm{~L}^{-1}$ & $263.4 \pm 61.9$ & $355.3 \pm 62.0$ & $165.5 \pm 22.5$ & $70.8 \pm 16.0$ \\
\hline \multicolumn{5}{|l|}{ Indolebutyric acid } \\
\hline $4.92 \times 10^{-4} \mathrm{~mol} \mathrm{~L}^{-1}$ & $214.6 \pm 01.3$ & $299.6 \pm 07.2$ & $119.4 \pm 04.9$ & $141.1 \pm 31.2$ \\
\hline $7.38 \times 10^{-4} \mathrm{~mol} \mathrm{~L}^{-1}$ & $361.1 \pm 46.3$ & $215.8 \pm 18.9$ & $305.6 \pm 26.4$ & $199.4 \pm 23.2$ \\
\hline $9.84 \times 10^{-4} \mathrm{~mol} \mathrm{~L}^{-1}$ & $214.1 \pm 06.6$ & $147.4 \pm 10.8$ & $131.5 \pm 17.7$ & $202.3 \pm 10.7$ \\
\hline \multicolumn{5}{|l|}{ Tryptophan } \\
\hline $4.89 \times 10^{-4} \mathrm{~mol} \mathrm{~L}^{-1}$ & $32.2 \pm 05.8$ & $166.0 \pm 04.6$ & $165.3 \pm 24.2$ & $47.3 \pm 09.6$ \\
\hline $7.34 \times 10^{-4} \mathrm{~mol} \mathrm{~L}^{-1}$ & $213.9 \pm 32.5$ & $293.1 \pm 35.7$ & $276.6 \pm 18.3$ & $193.2 \pm 20.1$ \\
\hline $9.79 \times 10^{-4} \mathrm{~mol} \mathrm{~L}^{-1}$ & $51.5 \pm 01.9$ & $22.3 \pm 04.2$ & $157.6 \pm 01.7$ & $23.7 \pm 0.22$ \\
\hline Distilled water & $98.5 \pm 21.3$ & $91.8 \pm 62.5$ & $45.4 \pm 07.4$ & $192.2 \pm 20.0$ \\
\hline Untreated & $262.4 \pm 12.2$ & $43.1 \pm 08.2$ & $162.7 \pm 31.6$ & $217.1 \pm 03.7$ \\
\hline \multicolumn{5}{|l|}{ LSD 5\% $=25.0$} \\
\hline & & & & \\
\hline \multicolumn{5}{|l|}{ Indoleacetic acid } \\
\hline $5.71 \times 10^{-4} \mathrm{~mol} \mathrm{~L}^{-1}$ & $66.5 \pm 10.7$ & $175.1 \pm 12.4$ & $57.8 \pm 07.0$ & $313.5 \pm 12.3$ \\
\hline $8.56 \times 10^{-4} \mathrm{~mol} \mathrm{~L}^{-1}$ & $105.9 \pm 13.7$ & $134.4 \pm 05.0$ & $88.2 \pm 09.3$ & $150.5 \pm 07.7$ \\
\hline $11.4 \times 10^{-4} \mathrm{~mol} \mathrm{~L}^{-1}$ & $60.9 \pm 05.5$ & $138.6 \pm 02.8$ & $90.6 \pm 11.6$ & $179.5 \pm 09.3$ \\
\hline \multicolumn{5}{|l|}{ Indolebutyric acid } \\
\hline $4.92 \times 10^{-4} \mathrm{~mol} \mathrm{~L}^{-1}$ & $152.9 \pm 12.4$ & $167.0 \pm 05.5$ & $111.5 \pm 16.9$ & $228.9 \pm 14.7$ \\
\hline $7.38 \times 10^{-4} \mathrm{~mol} \mathrm{~L}^{-1}$ & $73.9 \pm 08.3$ & $102.7 \pm 02.1$ & $65.6 \pm 15.3$ & $133.6 \pm 10.5$ \\
\hline $9.84 \times 10^{-4} \mathrm{~mol} \mathrm{~L}^{-1}$ & $98.6 \pm 11.5$ & $131.4 \pm 06.3$ & $105.4 \pm 06.4$ & $305.5 \pm 08.3$ \\
\hline \multicolumn{5}{|l|}{ Tryptophan } \\
\hline $4.89 \times 10^{-4} \mathrm{~mol} \mathrm{~L}^{-1}$ & $109.1 \pm 11.9$ & $251.7 \pm 19.5$ & $87.9 \pm 08.2$ & $195.9 \pm 12.5$ \\
\hline $7.34 \times 10^{-4} \mathrm{~mol} \mathrm{~L}^{-1}$ & $152.9 \pm 10.2$ & $139.0 \pm 03.9$ & $127.3 \pm 07.6$ & $127.8 \pm 09.1$ \\
\hline $9.79 \times 10^{-4} \mathrm{~mol} \mathrm{~L}^{-1}$ & $207.6 \pm 07.5$ & $195.3 \pm 08.9$ & $84.3 \pm 07.8$ & $209.5 \pm 05.6$ \\
\hline Distilled water & $119.2 \pm 01.6$ & $323.4 \pm 17.2$ & $93.2 \pm 07.0$ & $102.9 \pm 10.2$ \\
\hline Untreated & $96.4 \pm 08.6$ & $358.5 \pm 14.7$ & $162.1 \pm 11.8$ & $127.3 \pm 09.6$ \\
\hline
\end{tabular}

(1)LSD, least significant difference; distilled water, hydropriming; untreated, nonprimed seeds; IAA, indoleacetic acid; IBA, indolebutyric acid; ABA, abscisic acid. 
which caused reduction in free IAA concentration in leaves, in the salt-intolerant cultivar compared to the control.

Plants raised from IAA-primed $\left(11.42 \times 10^{-4} \mathrm{~mol} \mathrm{~L}^{-1}\right)$ seeds had higher IBA concentration in the salt-intolerant cultivar. In contrast, IBA $\left(4.92 \times 10^{-4} \mathrm{~mol} \mathrm{~L}^{-1}\right)$ increased free IAA concentration in the leaves, in the salt-stressed plants of the salt-tolerant cultivar. The differential behaviour might be due to differences in auxin-metabolism in the two cultivars. IAA is synthesized both from TRP (TRP-dependent pathway) and indolic TRP precursor (TRP-independent pathway). Even though IAA exists mostly in the form of IAA conjugates (Ludwig-Müller, 2011), salinity could alter IAA concentration in different plants (Javid et al., 2011). The homeostasis between IBA and IAA concentration has been shown to improve survival during drought and salt stress (Tognetti et al., 2010).
Plants of the salt-tolerant cultivar raised from auxin-primed seeds had higher free ABA in the leaves. However, the reverse was true in case of the salt-intolerant cultivar, in comparison with control. Both auxin and ABA act antagonistically to control/modulate different processes in plants (De Smet et al., 2003). Although pre-sowing seed treatments affected ABA levels differently (Table 2), they did not affect grain weight in both cultivars (Figure 2). However, priming with low concentrations (4.89$\left.7.34 \times 10^{-4} \mathrm{~mol} \mathrm{~L}^{-1}\right)$ of TRP increased fertile tillers per plant and, thus, effectively alleviated the drastic effect of salinity on grain yield, in both cultivars subjected to salt stress.

In the present study, plants of both cultivars raised from TRP-primed seeds had higher free putrescine (PUT), spermidine (SPD) and spermine (SPM) in leaves, in comparison with the control under salt stress (Figure 3).
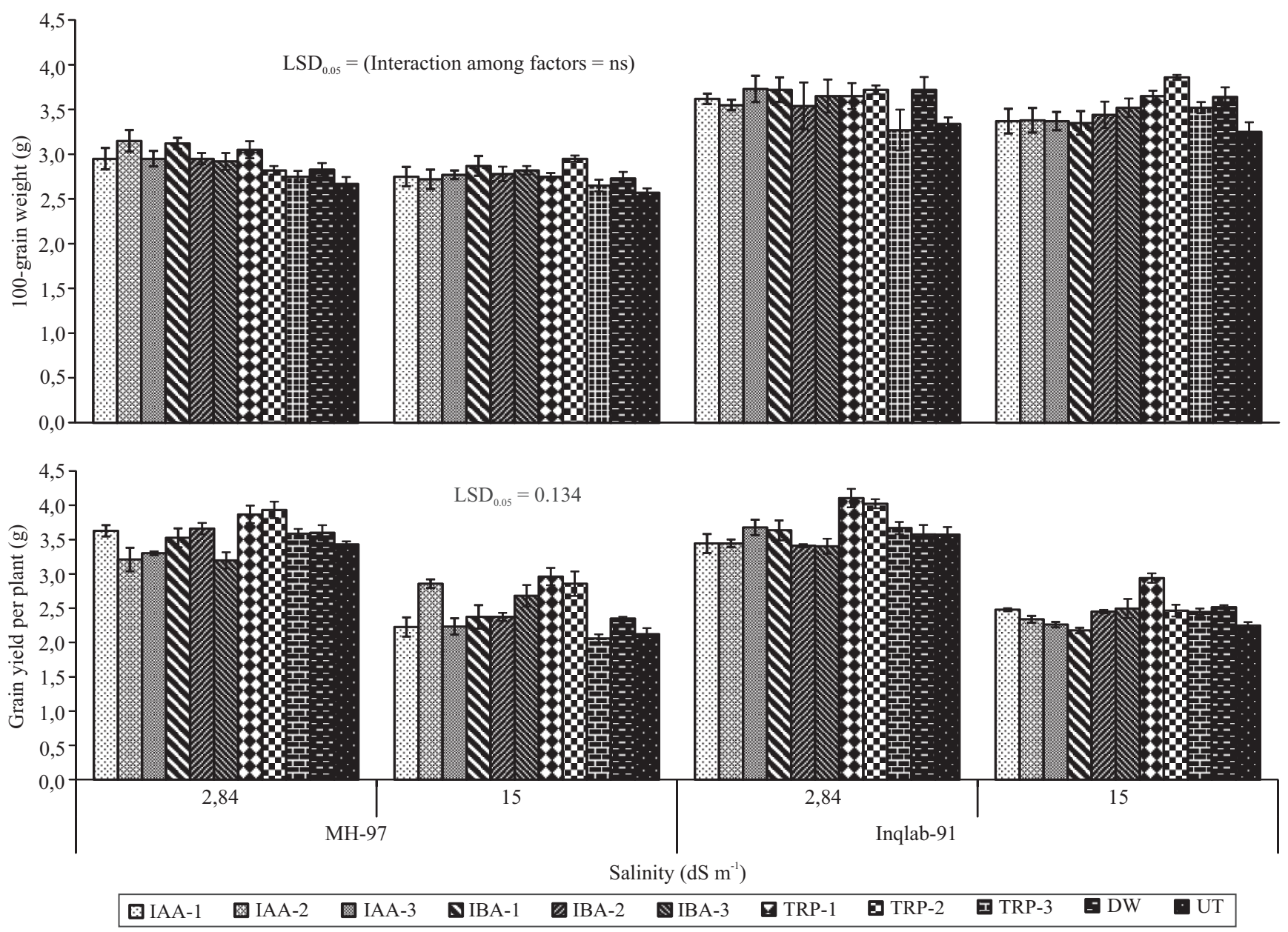

Figure 2. Grain weight and yield of wheat plants raised from primed seeds with auxins, or tryptophan, sown under both control $\left(2.82 \mathrm{dS} \mathrm{m}^{-1}\right)$ and saline $\left(15.0 \mathrm{dS} \mathrm{m}^{-1}\right)$ conditions. IAA-1-3, indoleacetic acid $\left(5.71,8.56\right.$ and $11.42 \times 10^{-4} \mathrm{~mol} \mathrm{~L}^{-1}$, respectively); IBA-1-3, indolebutyric acid (4.92, 7.38 and $9.84 \times 10^{-4} \mathrm{~mol} \mathrm{~L}^{-1}$, respectively); TRP-1-3, tryptophan (4.89, 7.34 and $9.79 \times 10^{-4} \mathrm{~mol} \mathrm{~L}^{-1}$, respectively); DW, soaking in distilled water, hydropriming; UT, untreated (nonprimed) seeds. 
The increase in SPM and a decrease in PUT in leaves suggested that TRP accelerated the conversion of PUT into SPD and SPM, which in turn was associated with better plant growth under salt stress. Plant capability to convert PUT into SPD and SPM closely relates to their developing ability under stress conditions (Chattopadhyay et al., 2002). The exogenously applied auxin could also alter polyamines transport (Bagni \& Pistocchi, 1991), thus affecting the tissue-specific polyamines concentrations.

Significant positive correlations of grain yield with different gas exchange characteristics, such as $\mathrm{A}(\mathrm{r}=0.466), \mathrm{g}_{\mathrm{s}}(\mathrm{r}=0.474)$ and $\mathrm{E}(\mathrm{r}=0.398)$, was observed in the present study (Table 3). Plant ability to increase growth and grain yield under salt stress was highly
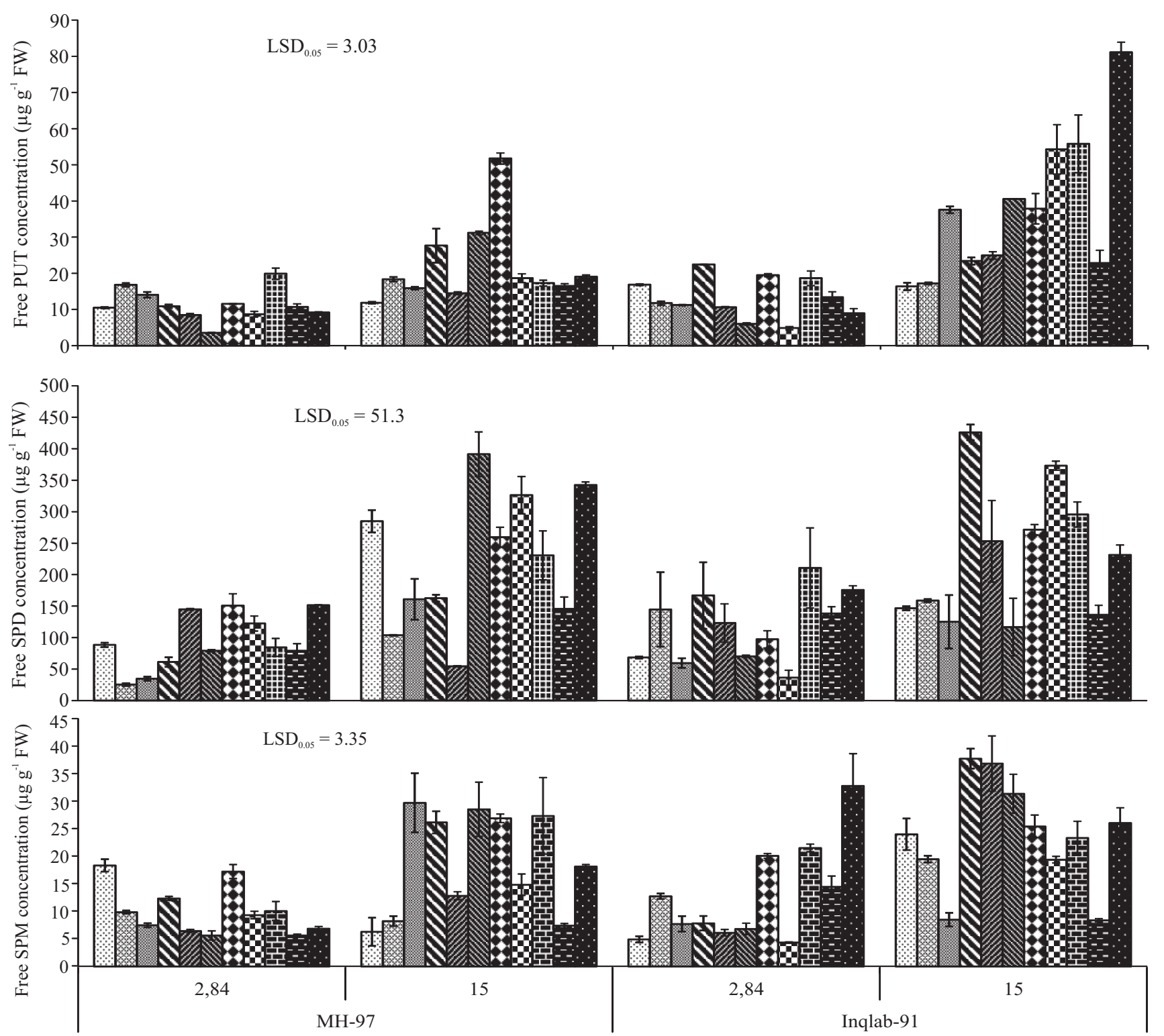

Salinity $\left(\mathrm{dS} \mathrm{m} \mathrm{m}^{-1}\right)$

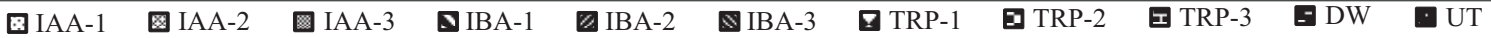

Figure 3. Leaf-concentrations of free putrescine (PUT), spermidine (SPD), and spermine (SPM) of wheat plants raised from primed seeds with auxins, or tryptophan, sown under both control $\left(2.82 \mathrm{dS} \mathrm{m}^{-1}\right)$ and saline $\left(15.0 \mathrm{dS} \mathrm{m}^{-1}\right)$ conditions. IAA-1-3, indoleacetic acid (5.71, 8.56 and $11.42 \times 10^{-4} \mathrm{~mol} \mathrm{~L}^{-1}$, respectively); IBA-1-3, indolebutyric acid (4.92, 7.38 and $9.84 \times 10^{-4} \mathrm{~mol} \mathrm{~L}^{-1}$, respectively); TRP-1-3, tryptophan $\left(4.89,7.34\right.$ and $9.79 \times 10^{-4} \mathrm{~mol} \mathrm{~L}^{-1}$, respectively); DW, soaking in distilled water, hydropriming; UT, untreated (nonprimed) seeds. 
Table 3. Pearson's correlation coefficient among grain yield, gas exchange characteristics, and hormonal concentrations of two spring wheat cultivars grown under both saline and nonsaline conditions.

\begin{tabular}{|c|c|c|c|c|c|c|c|c|c|c|}
\hline Variable $^{(1)}$ & GYP & $\mathrm{A}$ & $\mathrm{E}$ & gs & GW & IAA & IBA & ABA & PUT & SPD \\
\hline$\overline{\mathrm{A}}$ & $0.466^{*}$ & & & & & & & & & \\
\hline E & $0.398^{*}$ & $0.337^{*}$ & & & & & & & & \\
\hline gs & $0.474 *$ & $0.374 *$ & $0.324 *$ & & & & & & & \\
\hline GW & $0.217^{*}$ & 0.162 & $0.157^{*}$ & $0.212 *$ & & & & & & \\
\hline IAA & $0.519^{*}$ & 0.165 & $0.191^{*}$ & $0.171^{*}$ & -0.066 & & & & & \\
\hline IBA & 0.086 & 0.047 & 0.039 & $0.202 *$ & -0.125 & -0.087 & & & & \\
\hline $\mathrm{ABA}$ & $-0.514^{*}$ & $-0.252 *$ & $-0.195 *$ & $-0.390 *$ & $-0.204 *$ & $-0.296^{*}$ & $-0.352 *$ & & & \\
\hline PUT & $-0.442 *$ & -0.129 & $-0.373 *$ & $-0.207^{*}$ & 0.146 & $-0.388 *$ & $-0.167^{*}$ & $0.259 *$ & & \\
\hline SPD & $-0.464 *$ & -0.106 & $-0.237 *$ & $-0.296^{*}$ & -0.071 & $-0.369 *$ & -0.117 & $0.324 *$ & $0.417^{*}$ & \\
\hline SPM & $-0.398^{*}$ & -0.100 & $-0.183^{*}$ & $-0.181^{*}$ & 0.019 & $-0.297 *$ & -0.133 & $0.337 *$ & $0.431 *$ & $0.542 *$ \\
\hline
\end{tabular}

${ }^{(1)} \mathrm{GYP}$, grain yield per plant; A, net $\mathrm{CO}_{2}$ assimilation rate; E, transpiration rate; gs, stomatal conductance; GW, 100-grain weight; IAA, indoleacetic acid; IBA, indolebutyric acid; ABA, abscisic acid; PUT, putrescine; SPD, spermidine; and SPM, spermine. *Significant at $5 \%$ probability, by two-tailed test.

correlated with free IAA $(\mathrm{r}=0.519)$ in the leaves. In contrast, free $\mathrm{ABA}$ in the leaves showed a highly negative correlation $(\mathrm{r}=-0.514)$ with grain yield per plant, in both cultivars under salt stress. Endogenous concentrations of different polyamines (PUT, SPD and SPM) were negatively correlated $(\mathrm{r}=-0.442$, $\mathrm{r}=-0.464, \mathrm{r}=-0.398$, respectively) with grain yield. Thus, hormonal balance, rather than the real concentrations of hormones, played an important role in salt-tolerance of spring wheat.

\section{Conclusions}

1. Hormonal homeostasis is a key factor responsible for priming-induced salt-tolerance in spring wheat.

2. Auxin-priming-mediated hormonal homeostasis enhances $\mathrm{CO}_{2}$ assimilation rate, which results in better growth and higher grain yield, and confers tolerance to salinity in spring wheat.

3. Seed priming at low concentration of tryptophan was the most effective priming agent for minimizing losses in grain yield and assimilation rate due to salt stress, in genetically diverse spring wheat cultivars.

\section{References}

ASHRAF, M.Y.; AZHAR, N.; HUSSAIN, M. Indole acetic acid (IAA) induced changes in growth, relative water contents and gas exchange attributes of barley (Hordeum vulgare L.) grown under water stress conditions. Plant Growth Regulation, v.50, p.85-90, 2006. DOI: 10.1007/s10725-006-9130-6.

BAGNI, N.; PISTOCCHI, R. Uptake and transport of polyamines and inhibitors of polyamine metabolism in plants. In: SLOCUM, R.D.; FLORES, H.E. (Ed.). Biochemistry and physiology of polyamines in plants. Boca Raton: CRC Press, 1991. p.105-120.
CHATTOPADHYAY, S.; FARKYA, S.; SRIVASTAVA, A.K.; BISARIA, V.S. Bioprocess considerations for production of secondary metabolites by plant cell suspension cultures. Biotechnology and Bioprocess Engineering, v.7, p.138-149, 2002. DOI:10.1007/BF02932911.

CUARTERO, J.; BOLARIN, M.C.; ASINS, M.J.; MORENO, V. Increasing salt tolerance in the tomato. Journal of Experimental Botany, v.57, p.1045-1058, 2006. DOI:10.1093/jxb/erj102.

DE SMET, I.; SIGNORA, L.; BEECKMAN, T.; INZÉ, D.; FOYER, C.H.; ZHANG, H. An abscisic acid-sensitive checkpoint in lateral root development of Arabidopsis. Plant Journal, v.33, p.543-555, 2003. DOI:10.1046/j.1365-313X.2003.01652.x.

EL-BASSIOUNY, H.M.S. Physiological responses of wheat to salinity alleviation by nicotinamide and tryptophan. International Journal of Agriculture and Biology, v.4, p.653-659, 2005.

FINCH-SAVAGE, W.E.; DENT, K.C.; CLARK, L.J. Soak conditions and temperature following sowing influence the response of maize (Zea mays L.) seeds to on-farm priming (pre-sowing seed soak). Field Crops Research, v.90, p.361-374, 2004. DOI:10.1016/j.fcr.2004.04.006.

FLORES, H.E.; GALSTON, A.W. Analysis of polyamines in higher plants by high performance liquid chromatography. Plant Physiology, v.69, p.701-706, 1982. DOI:10.1104/pp.69.3.701.

FLOWERS, T.J. Improving crop salt tolerance. Journal of Experimental Botany, v.55, p.307-319, 2004. DOI: 10.1093/jxb/ erh003.

GRIEVE, C.M.; LESCH, S.M.; FRANCOIS, L.E.; MAAS, E.V. Analysis of main-spike yield components in salt-stressed wheat. Crop Science, v.32, p.697-703, 1992. DOI: 10.2135/cropsci1992. 0011183X003200030025x.

HERDE, O.; PEÑA-CORTÉS, H.; WILLMITZER, L.; FISAHN, J. Stomatal responses to jasmonic acid, linolenic acid and abscisic acid in wild-type and ABA-deficient tomato plants. Plant, Cell and Environment, v.20, p.136-141, 1997. DOI: 10.1046/ j.1365-3040.1997.d01-11.x.

IQBAL, M.; ASHRAF, M. Seed treatment with auxins modulates growth and ion partitioning in salt-stressed wheat plants. Journal 
of Integrative Plant Biology, v.49, p.1045-1057, 2007. DOI: 10.1111/j.1672-9072.2007.00488.x.

IQBAL, M.; ASHRAF, M.; JAMIL, A. Seed enhancement with cytokinins: changes in growth and grain yield in salt stressed wheat plants. Plant Growth Regulation, v.50, p.29-39, 2006. DOI: 10.1007/s10725-006-9123-5.

IQBAL, N.; NAZAR, R.; SYEED, S.; MASOOD, A.; KHAN, N.A. Exogenously-sourced ethylene increases stomatal conductance, photosynthesis, and growth under optimal and deficient nitrogen fertilization in mustard. Journal of Experimental Botany, v.62, p.4955-4963, 2011. DOI: 10.1093/jxb/err204.

JAMES, R.A.; RIVELLI, A.R.; MUNNS, R.; VON CAEMMERER, S. Factors affecting $\mathrm{CO}_{2}$ assimilation, leaf injury and growth in salt-stressed durum wheat. Functional Plant Biology, v.29, p.1393-1403, 2002. DOI: 10.1071/FP02069.

JAVID, M.G.; SOROOSHZADEH, A.; MORADI, F.; SANAVY, S.A.M.M.; ALLAHDADI, I. The role of phytohormones in alleviating salt stress in crop plants. Australian Journal of Crop Science, v.5, p.726-734, 2011.

KRUPINSKI, P.; JONSSON, H. Modeling auxin-regulated development. Cold Spring Harbor Perspectives in Biology, v.2, a001560, 2010. DOI: 10.1101/cshperspect.a001560.

KUMAR, B.; PANDEY, D.M.; GOSWAMI, C.L.; JAIN, S. Effect of growth regulators on photosynthesis, transpiration and related parameters in water stressed cotton. Biologia Plantarum, v.44, p.475-478, 2001. DOI: 10.1023/A:1012408624665.

KUSABA， S.; KANO-MURAKAMI, Y.; MATSUOKA, M.; TAMAOKI, M.; SAKAMOTO, T.; YAMAGUCHI, I.; FUKUMOTO, M. Alteration of hormone levels in transgenic tobacco plants over expressing the rice homeobox gene OSH1. Plant Physiology, v.116, p.471-476, 1998. DOI: 10.1104/ pp.116.2.471.

LIU, J.; MEHDI, S.; TOPPING, J.; FRIML, J.; LINDSEY, K. Interaction of PLS and PIN and hormonal crosstalk in Arabidopsis root development. Frontiers in Plant Science, v.4, 2013. DOI: 10.3389/fpls.2013.00075.

LUDWIG-MÜLLER, J. Auxin conjugates: their role for plant development and in the evolution of land plants. Journal of Experimental Botany, v.62, p.1757-1773, 2011. DOI: 10.1093/ $\mathrm{jxb} / \mathrm{erq} 412$.

LUDWIG-MÜLlER, J.; EPSTEIN, E.; HILGENBERG, W. Auxin-conjugate hydrolysis in Chinese cabbage: characterization of an amidohydrolase and its role during infection with the clubroot disease. Physiologia Plantarum, v.97, p.627-634, 1996. DOI: 10.1111/j.1399-3054.1996.tb00525.x.

MERRITT, F.; KEMPER, A.; TALLMAN, G. Inhibitors of ethylene synthesis inhibit auxin-induced stomatal opening in epidermis detached from leaves of Vicia faba L. Plant and Cell Physiology, v.42, p.223-230, 2001. DOI: 10.1093/pcp/pce030.

MUNNS, R. Genes and salt tolerance: bringing them together. New Phytologist, v.167, p.645-663, 2005. DOI: 10.1111/j.1469-8137.2 005.01487.x.

NORMANLY, J. Approaching cellular and molecular resolution of auxin biosynthesis and metabolism. Cold Spring Harbor Perspectives in Biology, v.2, a001594, 2010. DOI: 10.1101/ cshperspect.a001594.

POSPÍŠILOVÁ, J. Participation of phytohormones in the stomatal regulation of gas exchange during water stress. Biologia Plantarum, v.46, p.491-506, 2003. DOI: 10.1023/A:1024894923865.

ROBINSON, J.M. Does $\mathrm{O}_{2}$ photoreduction occur within chloroplast in vivo? Physiologia Plantarum, v.72, p.666-680, 1988. DOI: 10.1111/j.1399-3054.1988.tb09181.x.

SADEGHI, H.; NAZEMOSADAT, M.J. Effects of different levels of sodium chloride and photosynthetic photon flux density on some physiological traits in two wheat cultivars. African Journal of Agricultural Research, v.6, p.6326-6333, 2011. DOI: 10.5897/ AJAR09.586.

TANAKA, Y.; SANO, T.; TAMAOKI, M.; NAKAJIMA, N.; KONDO, N.; HASEZAWA, S. Cytokinin and auxin inhibit abscisic acid-induced stomatal closure by enhancing ethylene production in Arabidopsis. Journal of Experimental Botany, v.57, p.2259-2266, 2006. DOI: 10.1093/jxb/erj193.

TOGNETTI, V.; VAN AKEN, O.; MORREEL, K.; VANDENBROUCKE, K.; COTTE, B. van de; DE CLERCQ, I.; CHIWOCHA, S.; FENSKE, R.; PRINSEN, E.; BOERJAN, W.; GENTY, B.; STUBBS, K.; INZÉ, D.; VAN BREUSEGEM, F. Perturbation of indole-3-butyric acid homeostasis by the UDP-glucosyltransferase UGT74E2 modulates Arabidopsis architecture and water stress tolerance. Plant Cell, v.22, p.2660-2679, 2010. DOI: 10.1105/tpc.109.071316.

YEO, A.R.; FLOWERS, T.J. Salinity resistance in rice (Oryza sativa L.) and a pyramiding approach to breeding varieties for saline soils. Australian Journal of Plant Physiology, v.13, p.161-173, 1986. DOI: 10.1071/PP9860161.

Received on November 24, 2012 and accepted on August 19, 2013

Pesq. agropec. bras., Brasília, v.48, n.9, p.1210-1219, set. 2013 DOI: 10.1590/S0100-204X2013000900004 